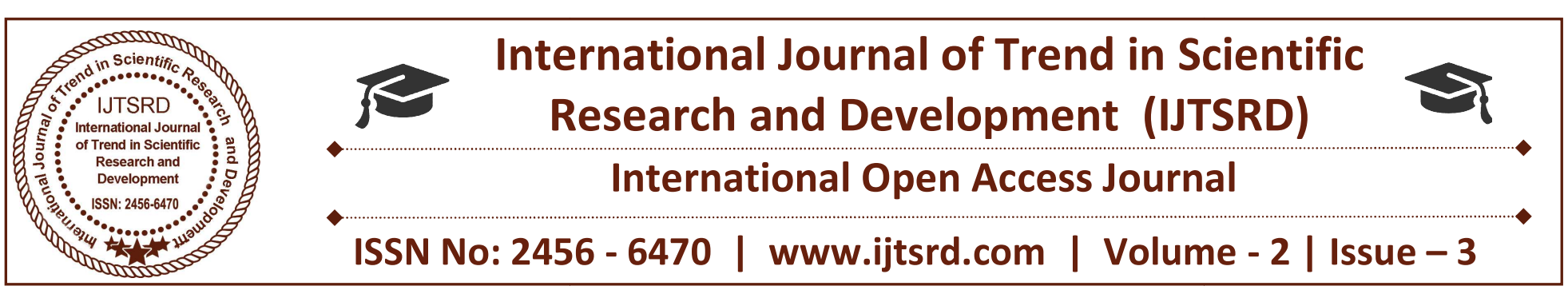

\title{
Optimum sizing methodologies and planning of different system components in hybrid power system
}

\author{
Reyaz Ahmed Bafanda \\ M.Tech Scholar, Electrical Engineering Department, \\ YIET, Gadhauli, Yamunanagar, Haryana, India
}

\begin{abstract}
In this paper a review is given related to the different sizing methodologies which can be opt in hybrid power generation system incorporating renewable energy resources (RERs). Different criteria of sizing the different system components of hybrid renewable energy power plant at the most preferable logistical environmental and economical considerations have been discussed. Also, the paper discussed some of the optimization approaches which are used to compare the energy production cost and performance of different hybrid system configurations e using HOMER.
\end{abstract}

\section{INTRODUCTION}

Hybrid Renewable Energy Systems (HRES) are defined as an electric energy system which is made up of one renewable source and one or more sources. These sources could be conventional or renewable or mixed, that works in off-grid (standalone) or grid connected mode [1]. The main feature of hybrid renewable energy systems is to combine two or more renewable power generation and so they can address emissions, reliability, efficiency, and economical limitations of single renewable energy source [2].

HRES are becoming popular for standalone power generation in isolated area due to the improvement and efficiency increment in renewable energy technologies and power electronic converters [3]. Based on the availability of the natural local resources, there are some advantages of the hybrid system. Higher environmental protection, green house gas emission, especially $\mathrm{CO} 2$ and reduction in other pollutants emissions is expected due to the lower consumption of fuel. The cost of solar and wind energy can be competitive with nuclear and the diversity and security of natural resources who are free, abundant, and inexhaustible [4]. Most of these appliances can be easily installed and they are rapidly deployed. Financially, the costs are predictable and not influenced by fuel price fluctuations [5-8]. However, because of the photovoltaic (PV)-Wind changeable nature and dependence on climatic changes and weather, a common drawback to PV and wind power generations is that both would have to be oversized to make their standalone systems reliable for the times when neither system is producing enough electric power to satisfy the load [9].

Many areas are concerned with the applications of the HRES. Researches $[1,10]$ have focused on the performance analysis of demonstration systems and the development of efficient power converters, such as the maximum power point trackers and bidirectional inverters $[11,12]$. Other researches focused on the battery management units and the storages devices [6].

In the last decade, various HRES have been installed in different countries, resulting in the development of systems that can compete with conventional, fuel based remote area power supplies [13]. However, there are several combinations of hybrid energy system which mainly depend on the natural available resources, the wind or the solar energy practically represents one source of the hybrid renewable energy systems.

With the advance development of the hybrid PVWind systems for electrical power generation, the target to achieve reliable and efficient performance 
became complicated task. So that there is a need to select and configure the optimum sizing of all components in order to obtain the minimum capital investment while maintaining system reliability to satisfy the load $[14,15]$. This paper summarized three common used sizing methods for hybrid PV-Wind systems. Moreover, the paper will discuss some optimization techniques of the HRES. These techniques are used to compare the technical performance and cost of energy for different system configurations using simulation approaches.

One of the most powerful tools for optimal sizing of HRESs' equipment is Hybrid Optimization Model for Electric Renewables (HOMER) software that was developed by National Renewable Energy Laboratory (NREL), United States [16]. Although HOMER software is used in many studies. Therefore, an article is needed that comprehensively reviews the papers which used HOMER for optimal planning of HRESs which is the main objective of this paper. This review will be useful for researchers who intend to use HOMER for planning of HRES in their regions. It provides the required information about planning of HRES simulated with HOMER such as what components are considered in HRESs? How they are used in stand-alone or grid connected modes? And what uncertain parameters are considered in the articles?

\section{DESCRITION OF HOMER SOFTWARE}

HOMER software is a powerful tool for designing and planning of HRES in order to determine optimal size of its components through carrying out the technoeconomic analysis. Many resources such as WT, PV array, fuel cells, small hydropower, biomass, converter, batteries, and conventional generators are modeled in HOMER. HOMER also considers HRES in grid-connected and stand-alone modes. Fig. 1 shows the typical configuration of HRES designed in HOMER. Required input data for simulation with HOMER and also a comprehensive framework to show how optimal sizes of HRES's equipment is determined by HOMER are described in this section.

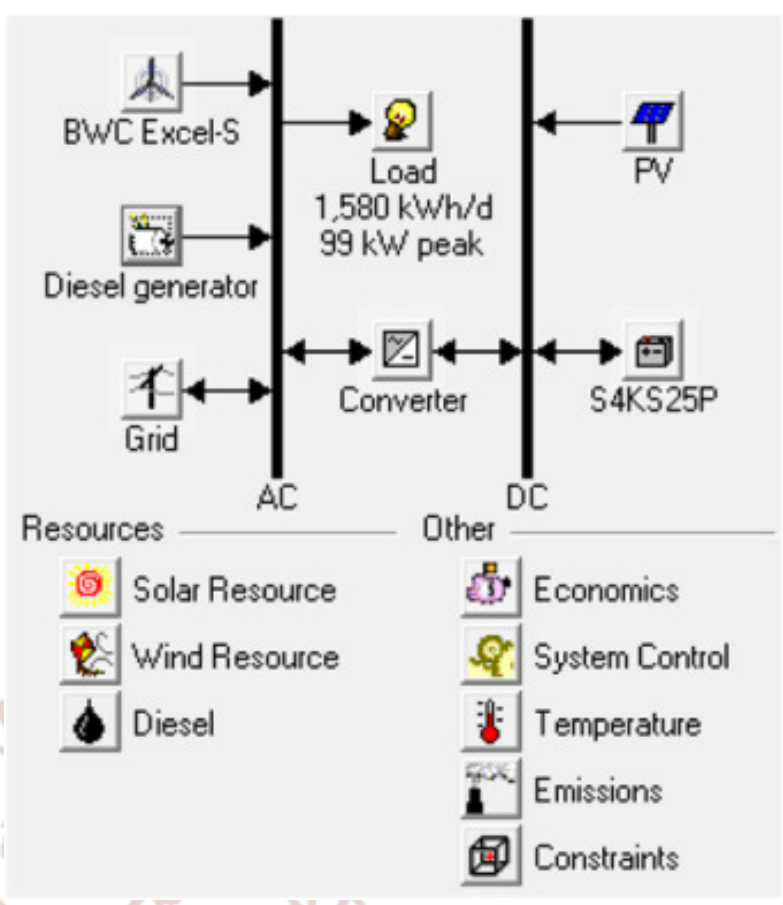

Fig. 1. Typical schematic of a HRES in HOMER

I. Input data

HOMER requires six types of data for simulation and optimization including meteorological data, load profile, equipment characteristics, search space, economic and technical data. These data are described in details in the following subsections.

a. Meteorological data

The meteorological data are wind speed, solar radiation, temperature, and stream flow which are fed into the software in the form of monthly averages or time series data. HOMER uses these inputs data to calculate the output power of WT, PV array and hydropower.

b. Load profile

Load profile of each region is the most important factor in the simulation and optimization. Some locations such as universities, hospitals, hotels, and industrial towns have real load consumption data, which are appropriate for simulation. These real data are fed into HOMER as time series data. However, in some regions especially remote and rural areas that the real load consumption data are not available, the load profile should be forecasted with notice to the specification of that region. These data are fed into HOMER as daily profile and HOMER uses them in power balance constraint.

c. Equipment characteristics

According to the characteristics of each equipment, which is modeled in HOMER, efficient operation of it 
in HRES is determined. The characteristics of HRES's equipment are described in [11].

\section{d. Search space}

Since HRESs' components including WT, PV array, generator, battery, and converter have different sizes, there is a search space that is considered in simulation and optimization. For example, the equipment of a hypothetical HRES which have different sizes is illustrated in Table 1 . So, the search space includes $5 \times 5 \times 5 \times 3=375$ plans (combination of different equipment) that the simulation and optimization stages will be done for each of them.

Table 1. Search space for a hypothetical case study

\begin{tabular}{|l|l|l|l|l|}
\hline Component & $\begin{array}{l}\text { WT } \\
(\text { number })\end{array}$ & $\begin{array}{l}\text { PV } \\
\text { array } \\
(\mathrm{kW})\end{array}$ & $\begin{array}{l}\text { Battery } \\
\text { (number) }\end{array}$ & $\begin{array}{l}\text { Converter } \\
(\mathrm{kW})\end{array}$ \\
\hline Maximum & 4 & 40 & 40 & 40 \\
\hline Minimum & 0 & 0 & 0 & 0 \\
\hline Step & 1 & 10 & 10 & 20 \\
\hline
\end{tabular}

e. Economic data

Each equipment in HRES has some cost data such as operation and maintenance, capital, and replacement cost. Fuel price, price of transaction electricity with the grid, real interest rate, project lifetime, system fixed capital cost, system fixed operation and maintenance cost, and emissions penalty are the other economic data that can be considered in HOMER. These costs are considered in simulation and optimization stages and based on them, the Net Present Cost (NPC) of each plan is calculated.

\section{f. Technical data}

For simulation, HOMER requires some technical data including dispatch strategy, MACS, MREF, and operating reserve, which are described in [11].

\section{g. HOMER optimization procedure}

After the input data are fed into HOMER, which was described in previous section, optimal sizes of HRES's equipment are determined in three stages including simulation, optimization, and sensitivity analysis as described in Fig. 2. These stages are introduced in the following subsections. Simulation and optimization stages are done simultaneously.

\section{Simulation and optimization}

For each plan from search space the optimization and simulation stages are done. The objective function is minimized subject to the constraints. The objective function of each plan is the total NPC which is the present value of the sum of costs minus the sum of revenues. The costs are the cost of energy purchased from the grid, initial cost, replacement cost, operation and maintenance cost as well as the fuel cost. The revenues are the revenue from energy sold to the grid and the salvage value. Constraints are power balance constraint, charging and discharging constraints of battery, constraints of transaction energy with grid, technical constraints of generators and so on. For the feasible plans the required output is calculated including the NPC, operation results of resources such as generator, battery, and converter in each time step, the energy transacted with the grid, and the emissions produced. Feasibility means that the power balance constraint is satisfied in each time step. In fact, the demand of each time step is supplied. At the end, the feasible plans are sorted according to the minimum NPC so that the first plan has the minimum NPC and is considered as the best plan.

a. Sensitivity analysis

In optimal sizing procedure of HRES's equipment, some parameters such as fuel cost, wind speed, solar radiation, electricity price, and components cost have not deterministic values. So, the uncertainty of these parameters has effect on simulation and optimization stages. These parameters are fed into HOMER with different values. When the simulation and optimization stages are done and the feasible plans are sorted according to the minimum NPC, the sensitivity analysis is done as shown in Fig. 2. For each uncertain parameter the simulation and optimization stages are repeated and the new feasible and best plans may be obtained. To evaluate the effect of uncertain parameters on the results, two criteria including robust planning and risk of planning are proposed in $[12,13]$. These criteria can be calculated by the researchers according to the methodologies which are described in $[12,13]$ and HOMER software is not able to calculate them. HOMER also produces appropriate figures to show how the best plans' output will be changed with uncertain parameters.

\section{Equipment modeled in HOMER}

In this section, various HRES's equipment modeled in HOMER and used in different articles is presented. Loads, components, and grid are three types of the HRES's equipment, which are modeled in HOMER.

a. Loads

HRES should meet the load requirements in each time step. Electrical, thermal, and hydrogen loads are 
modeled in HOMER. Electrical loads are primary and deferrable loads. Primary loads are the electrical load that must be met in certain time while deferrable load is the electrical load that must be met within some time period, but the exact time is not important. Peak and types of different loads that are used in different articles are listed in Table 2. Also, Table 2 shows the locations and countries which are considered in each article. Locations that are listed for each article, such as remote area, rural, and village are exactly mentioned in the same article.

\section{b. Components}

In HOMER, each part of HRES that can produce, deliver, convert, or save energy is named as a component. Ten components are modeled in the HOMER. WT, PV, and small hydropower are three renewable energy and non-dispatchable resources. Generators, grid, and boiler are three dispatchable resources. Converter and electrolyzer are components that convert electrical energy to other forms. AC and DC power are converted to each other using converters and electrolyzers consume $\mathrm{AC}$ or $\mathrm{DC}$ power and generate hydrogen through electrolyzing water. Batteries and hydrogen tanks are components that store energy. HRES that are modeled in the articles have used different components for simulation.

\section{c. Grid}

Grid is modeled in the HOMER in three modes, namely, grid-connected, stand-alone, and compare stand-alone system with grid extension. Table 4 shows different modes of HRESs used in the articles. In grid connected mode price and sell back of electricity should be fed into HOMER in two types, real time prices and scheduled rates. In compare stand-alone system with grid extension mode, breakeven grid extension distance will be calculated using three inputs including capital, operation and maintenance cost and grid power price. The breakeven grid extension distance is the minimum distance from the grid that makes a stand-alone system cheaper than extending the grid.

\section{SENSITIVITY ANALYSIS}

To evaluate the effect of uncertain parameters on optimal sizing of HRES's equipment, sensitivity analysis should be done. Uncertain parameters may differ with notice to the location and type of the components. Wind speed, solar radiation, fuel price, component cost, and primary load are the most uncertain parameters considered in the researches.
In most articles the effect of uncertain parameters on NPC, operation results of resources, production of emissions and other parameters of best plans are evaluated. Moreover, sensitivity analysis is done in details in recent papers. Effects of real interest rate, fuel price, and primary load on output results is investigated through different scenarios. The simultaneous effects of wind speed and solar radiation on $\mathrm{CoE}$. To investigate the effect of uncertain parameters on the NPC, an appropriate spider graph is presented. However, as mentioned before, to evaluate uncertain parameters and their effect on optimal sizing of HRES's equipment, two appropriate criteria including robust planning and risk of planning should be considered. Scenario technique is one of the appropriate approaches in dealing with the uncertainties in planning of HRES. To this end, at first, uncertain parameters and their different values that may occur in the future is determined and based on them, the scenarios can be constructed. For example, in one project the uncertain parameters and their different values may be considered.

\section{DISCUSSION ON HOMER}

After all required data are fed into HOMER and the simulation and optimization stages are done, the results of each plan including the NPC (\$), the initial capital cost (\$), the operation cost $(\$ / y r)$, renewable fraction (percent), $\mathrm{CoE}(\$ / \mathrm{kWh})$, and emissions produced $(\mathrm{kg} / \mathrm{yr})$ are calculated. Although best plan is determined according to the minimum NPC by HOMER, the best plan may be selected with notice to the other criteria considering the investors' perspective.

$\mathrm{CoE}$ is an appropriate criterion to choice the best plan that is used in the literature. This criterion indicates the average cost per kWh of each plan to supply the demand. The CoE is high in some cases due to high investment cost of component, high fuel prices, high distance from the main grid and so on. For electrification to remote and rural areas which have high distance from the main grid, there are two main solutions including the extension of the main grid and using of HRESs. Although extension of the main grid may lead to lower NPC in comparison with HRESs, it has several disadvantages including power losses, low power quality, and high operation and maintenance cost. Moreover, in some cases the grid extension in not possible with notice to the topography of region. On the other hand, in urban areas the CoE for best plans is higher than the grid electricity prices. Therefore, to encourage the private investors to invest 
on HRESs in rural and urban areas, the governments should determine attractive regulations to give more incentives to investors.

\section{Conclusions}

HRESs are appropriate solution to meet the local loads in rural, remote, and special urban regions, e.g., universities and hospitals. Determining the optimal sizes of HRES's equipment is the major concern of researchers. HOMER software is a powerful tool used by many researchers around the world for optimal planning of HRES. According to the ability and widespread use of this software, the present paper reviewed those articles that have used HOMER for the optimal planning of HRES. The most remarkable conclusions from this review are listed as follows:

$>$ The software has been used in developing countries more than other regions, especially in remote and rural areas.

$>$ The software has been used for wide range of load from $0.626 \mathrm{~kW}$ to $2,213,000 \mathrm{~kW}$.

$>$ Many combinations of dispatchable/Nondispatchable resources, storages and converters have been modeled in the articles.

$>\mathrm{PV}$ is the popular resource considered by many researchers.

HRESs have been modeled in stand-alone mode more than grid connected mode.

\section{References}

1) Erdinc O, Uzunoglu/M. Optimum design of hybrid renewable energy systems: overview of different approaches. Renew Sustain Energy Rev 2012;16:1412-25.

2) Bajpai P, Dash V. Hybrid renewable energy systems for power generation in stand-alone applications: A review. Renew Sustain Energy Rev 2012;16:2926-39.

3) Bernal-Agustín JL, Dufo-López R. Simulation and optimization of stand-alone hybrid renewable energy systems. Renew Sustain Energy Rev 2009;13:2111-8.

4) Bhattacharyya SC. Review of alternative methodologies for analysing off-grid electricity supply. Renew Sustain Energy Rev 2012;16:67794.

5) Fathima AH, Palanisamy K. Optimization in microgrids with hybrid energy systems - a review. Renew Sustain Energy Rev 2015;45:431-46.

6) Kaundinya DP, Balachandra P, Ravindranath $\mathrm{NH}$. Grid-connected versus stand-alone energy systems for decentralized power - a review of literature. Renew Sustain Energy Rev 2009;13:2041-50.

7) Mahesh A, Sandhu KS. Hybrid wind/photovoltaic energy system developments: critical review and findings. Renew Sustain Energy Rev 2015;52:1135-47.

8) Shivarama Krishna K, Sathish Kumar K. A review on hybrid renewable energy systems. Renew Sustain Energy Rev 2015;52:907-16.

9) Upadhyay S, Sharma MP. A review on configurations, control and sizing methodologies of hybrid energy systems. Renew Sustain Energy Rev 2014;38:47-63.

10) Lambert T, Gilman P, Lilienthal P. Micropower system modeling with homer. Integration of alternative sources of energy. John Wiley \& Sons, Inc.; 2006. p. 379-418.

11) Andrews CJ. Evaluating risk management strategies in resource planning. IEEE Trans Power Syst 1995;10:420-6.

12) Laimes YY. Risk modeling, assessment, and management. John Wiley \& Sons; 2005.

13) Blackler T, Iqbal MT. Pre-feasibility study of wind power generation in Holyrood, Newfoundland. Renew Energy 2006;31:489-502.

14) Khan MJ, Iqbal MT. Pre-feasibility study of stand-alone hybrid energy systems for applications in Newfoundland. Renew Energy 2005;30:835-54.

15) Nfah EM, Ngundam JM, Vandenbergh M, Schmid J. Simulation of off-grid generation options for remote villages in Cameroon. Renew Energy 2008;33:1064-72.

16) $\langle$ http://www.nrel/gov/HOMER〉. 